\title{
IOAN TOMESCU
}

\section{An upper bound for the shortest hamiltonian path in the symmetric euclidean case}

Revue française d'automatique, d'informatique et de recherche opérationnelle. Recherche opérationnelle, tome 17, n 3 (1983), p. 297-306.

<http://www.numdam.org/item?id=RO_1983_17_3_297_0>

(C) AFCET, 1983, tous droits réservés.

L'accès aux archives de la revue « Revue française d'automatique, d'informatique et de recherche opérationnelle. Recherche opérationnelle » implique l'accord avec les conditions générales d'utilisation (http://www.numdam.org/ legal.php). Toute utilisation commerciale ou impression systématique est constitutive d'une infraction pénale. Toute copie ou impression de ce fichier doit contenir la présente mention de copyright.

\section{Numdam}

Article numérisé dans le cadre du programme

Numérisation de documents anciens mathématiques

http://www.numdam.org/ 


\title{
AN UPPER BOUND FOR THE SHORTEST HAMILTONIAN PATH IN THE SYMMETRIC EUCLIDEAN CASE (*)
}

\author{
by Ioan Tomescu $\left({ }^{1}\right)$
}

\begin{abstract}
In this paper an algorithm for obtaining a Hamiltonian path from a shortest spanning tree of a complete weighted graph is proposed. As a consequence, two inequalities between the costs of a shortest Hamiltonian path and a shortest spanning tree $T$ in the symmetric Euclidean case are proposed. These inequalities involve the diameter of $T$ or the number of terminal vertices of $T$ and they become equalities in some particular cases.
\end{abstract}

Keywords: Hamiltonian path, Spanning tree, Triangle inequality.

Résumé. - Dans ce travail on propose un algorithme pour l'obtention d'une chaîne hamiltonienne en partant de l'arbre minimal d'un graphe complet non orienté valué. Comme conséquence l'on déduit une borne du coût d'une chaîne hamiltonienne minimale dans le cas où les coûts satisfont l'inégalité du triangle. Cette borne fait intervenir le diamètre et le nombre des sommets pendants de T, elle devient une égalité dans certains cas particuliers.

Mots clés : Chaîne hamiltonienne, arbre partiel, inégalité du triangle.

\section{DEFINITIONS AND NOTATION}

Let $K_{n}$ be the complete graph having vertex set $V\left(K_{n}\right)=\{1, \ldots, n\}$ and edge set $E\left(K_{n}\right)=\{i j \mid 1 \leqq i<j \leqq n\}$.

To each edge $i j$ is associated a cost $c(i, j)=c_{i j} \geqq 0$ such that:

$$
C=\left(c_{i j}\right)_{i, j=1, \ldots, n}
$$

is a symmetric matrix having $c_{i i}=0$ on the main diagonal.

Suppose also that elements of $C$ verify triangle inequality:

$$
c_{i j} \leqq c_{i k}+c_{k j}
$$

for any $i, j, k=1, \ldots, n$, i. e. $c_{i j}$ may be considered as a distance between vertices $i$ and $j$ of $K_{n}$.

(*) Reçu février 1982.

(1) Faculté de Mathématiques, Université de Bucarest, Str. Academiei, 14, 70108 București, Roumanie.

R.A.I.R.O. Recherche opérationnelle/Operations Research, 0399-0559/1983/297/\$5.00

(C) AFCET-Bordas-Dunod 
The cost of a path (cycle) of $K_{n}$ is equal to the sum of the costs of the edges of this path (cycle).

A shortest spanning tree (SST) of $K_{n}$ is a spanning tree of $K_{n}$ having minimum cost. In a similar way a shortest Hamiltonian path (SHP) and a shortest Hamiltonian cycle (SHC) are defined.

The problem of determining a shortest Hamiltonian cycle of $K_{n}$ is also known as the travelling salesman problem [4].

Some connections between SST's problem and SHC's problem were discovered by $\mathbf{M}$. Held and R. M. Karp [5].

Using a similar method of penalizing vertices, $\mathrm{N}$. Christofides proposed an algorithm for solving SHP's problem which starts from a SST of $K_{n}$ [3]. This algorithm is not necessarily convergent and it produces a variety of spanning trees of $K_{n}$ associated to matrices of costs preserving all SHP having two fixed extremities. I. Tomescu proposed an algorithm for obtaining a Hamiltonian path (HP) of $K_{n}$ [7] which is based on 3 types of transformations of a SST, denoted respectively by 1,2 and 3 .

These transformations are used in order to decrease at each step the number of terminal vertices (or leaves) of the tree, until it is obtained a spanning tree of $K_{n}$ having exactly two terminal vertices, which is a HP of $K_{n}$.

No estimation is made in [7] about the error of the algorithm for obtaining a SHP, even in the Euclidean symmetric case, when the costs satisfy triangle inequality.

In the sequel we shall obtain such an evaluation if only transformations of type 3 are applied.

For a spanning tree $T$ of $K_{n}$ we shall define the distance $d_{T}(i, j)$ between vertices $i$ and $j$ by the cost of the unique path of $T$ joining $i$ and $j$ and the diameter of $T$, denoted by $\operatorname{diam}(T)$, as

$$
\operatorname{diam}(T)=\max _{1 \leqq i, j \leqq n} d_{T}(i, j)
$$

We shall denote by $c$ (SST), $c$ (SHP), $c$ (HP), $c$ (SHC), the cost of a SST, SHP, HP, respectively SHC of $K_{n}$.

The degree of a vertex $x$ in a tree $T$ is denoted $\operatorname{deg}_{T}(x)$.

An elementary subdivision of a nonempty graph $G$ is a graph obtained from $G$ by the removal of some edge $e=u v$ and the addition of a new vertex $w$ and edges $u w$ and $v w$.

A subdivision of $G$ is a graph obtained from $G$ by a succession of elementary subdivisions [1]. 


\section{UPPER BOUNDS FOR $c$ (SHP)}

Suppose now that $T$ is a SST of $K_{n}$ having $t(T)=t$ terminal vertices. If $t=2$ then $T$ is a SHP of $K_{n}$. Otherwise, $t \geqq 3$ and we shall describe an algorithm which transforms $T$ into a Hamiltonian path of $K_{n}$, denoted HP, such that:

$$
c(\mathrm{HP}) \leqq 2 c(T)-\operatorname{diam}(T) .
$$

For this, let $i, j$ be two terminal vertices of $T$ such that $d_{T}(i, j)=\operatorname{diam}(T)$. If $x$ is a terminal vertex of $T, x \neq i, j$, there exists a unique path $P: x, x_{1}, x_{2}, \ldots, x_{p}$ of $T, \quad p \geqq 1, \quad$ such that $\operatorname{deg}_{T}\left(x_{1}\right)=\operatorname{deg}_{T}\left(x_{2}\right)=\ldots=\operatorname{deg}_{T}\left(x_{p-1}\right)=2$ and $\operatorname{deg}_{T}\left(x_{p}\right) \geqq 3$. There is a vertex $v$ adjacent to $x_{p}$ such that $v \notin V(P)$.

From $T$ we obtain two trees $T_{1}$ and $T_{1}^{\prime}$ defined as follows:

$T_{1}$ is obtained from $T$ by deleting vertices $x, x_{1}, \ldots, x_{p-1}$ and their incident edges;

$T_{1}^{\prime}$ is obtained from $T$ by deleting edge $x_{p} v$ and inserting new edge $x v$, hence $V\left(T_{1}^{\prime}\right)=V(T)=\{1, \ldots, n\}$.

It is clear that $T_{1}^{\prime}$ is isomorphic to a graph obtained from $T_{1}$ by inserting $p$ new vertices on the edge $x_{p} v$, or $T_{1}^{\prime}$ is a subdivision of $T_{1}$ and $t\left(T_{1}\right)=t\left(T_{1}^{\prime}\right)=t-1$.

We can write:

$$
c\left(T_{1}^{\prime}\right)=c(T)-c\left(x_{p}, v\right)+c(x, v)
$$

By applying triangle inequality we derive:

$$
\begin{aligned}
c(x, v) \leqq c\left(x, x_{1}\right)+c\left(x_{1}, v\right) \leqq c\left(x, x_{1}\right) & \\
+c\left(x_{1}, x_{2}\right)+c\left(x_{2}, v\right) \leqq & \ldots \leqq c\left(x, x_{1}\right)+c\left(x_{1}, x_{2}\right)+\ldots \\
& +c\left(x_{p-1}, x_{p}\right)+c\left(x_{p}, v\right)=d_{T}\left(x, x_{p}\right)+c\left(x_{p}, v\right) .
\end{aligned}
$$

From (1) we deduce that:

$$
c\left(T_{1}^{\prime}\right) \leqq c(T)+d_{T}\left(x, x_{p}\right)=c(T)+c(P)
$$

If $t=3$ then $t\left(T_{1}\right)=t\left(T_{1}^{\prime}\right)=2$ and we define $\mathrm{HP}=T_{1}^{\prime}$.

Otherwise, let $y$ be a terminal vertex of $T_{1}, y \neq i, j$ and the path:

$$
P_{1}: y, y_{1}, y_{2}, \ldots, y_{q}
$$

of $T_{1}, q \geqq 1$, such that:

$$
\operatorname{deg}_{T_{1}}\left(y_{1}\right)=\operatorname{deg}_{T_{1}}\left(y_{2}\right)=\ldots=\operatorname{deg}_{T_{1}}\left(y_{q-1}\right)=2 \text { and } \operatorname{deg}_{T_{1}}\left(y_{q}\right) \geqq 3 \text {. }
$$

vol. $17, \mathrm{n}^{\circ} 3$, août 1983 
Since $T_{1}^{\prime}$ is a subdivision of $T_{1}$ it follows that all terminal vertices and all vertices of degree greater than 2 are the same in $T_{1}$ and $T_{1}^{\prime}$.

Hence we derive that there is a path $Q_{1}$ between $y$ and $y_{q}$ in $T_{1}^{\prime}$ such that all vertices of this path different from $y$ and $y_{q}$ have their degrees in $T_{1}^{\prime}$ equal to 2. But $\operatorname{deg}_{T_{1}^{\prime}}\left(y_{q}\right) \geqq 3$ implies that there exists a vertex $w$ adjacent to $y_{q}$ in $T_{1}^{\prime}$ such that $w \notin V\left(Q_{1}\right)$. From $T_{1}$ we obtain a new tree $T_{2}$ and from $T_{1}^{\prime}$ a tree $T_{2}$ which are defined as follows:

$T_{2}$ is deduced from $T_{1}$ by the removal of vertices $y, y_{1}, \ldots, y_{q-1}$ and their incident edges and $T_{2}^{\prime}$ is obtained from $T_{1}^{\prime}$ by deleting edge $y_{q} w$ and inserting new edge $y w$, hence $V\left(T_{2}^{\prime}\right)=\{1, \ldots, n\}$.

$T_{2}^{\prime}$ is a subdivision of $T_{2}$ and $t\left(T_{2}\right)=t\left(T_{2}^{\prime}\right)=t-2$.

We derive also:

$$
c\left(T_{2}^{\prime}\right)=c\left(T_{1}^{\prime}\right)-c\left(\gamma_{q}, w\right)+c(y, w)
$$

By applying triangle inequality we get:

$$
\begin{gathered}
c(y, w) \leqq c\left(y, y_{1}\right)+c\left(y_{1}, w\right) \leqq \ldots \\
\leqq c\left(y, y_{1}\right)+c\left(y_{1}, y_{2}\right)+\ldots+c\left(y_{q-1}, y_{q}\right)+c\left(y_{q}, w\right) \\
=d_{T_{1}}\left(y, y_{q}\right)+c\left(y_{q}, w\right)=d_{T}\left(y, y_{q}\right)+c\left(y_{q}, w\right),
\end{gathered}
$$

since $T_{1}$ is a subtree of $T$.

Hence (3) implies:

$$
c\left(T_{2}^{\prime}\right) \leqq c\left(T_{1}^{\prime}\right)+d_{T}\left(y, y_{q}\right)=c\left(T_{1}^{\prime}\right)+c\left(P_{1}\right) .
$$

If $t=4$ then $T_{2}^{\prime}$ is a Hamiltonian path and we define $\mathrm{HP}=T_{2}^{\prime}$. Otherwise, we consider a terminal vertex $z \neq i, j$ of $T_{2}$ and we obtain in a similar manner trees $T_{3}$ and $T_{3}^{\prime}$ and so on.

Note that $T_{i}$ are subtrees of $T$ containing all the $i-j$ path of $T$ whereas $T_{i}^{\prime}$ are spanning trees of $K_{n}$, as is illustrated in figure 1 .

It follows that $T_{t-2}$ is the unique path $P_{t-2}$ of $T$ joining vertices $i$ and $j$ and $T_{t-2}^{\prime}$ is a Hamiltonian path of $K_{n}$. We define $\mathrm{HP}=T_{t-2}^{\prime}$. From inequalities (2), (4), ... we deduce that:

$$
c(\mathrm{HP})=c\left(T_{t-2}^{\prime}\right) \leqq c(T)+\sum_{i=0}^{t-3} c\left(P_{i}\right),
$$

where $P_{0}=P$. 


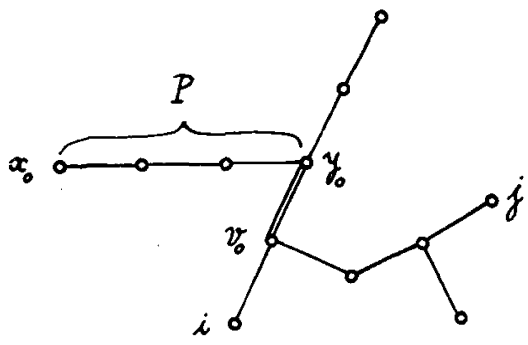

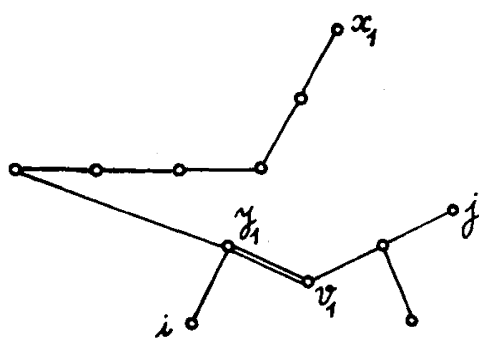

$T_{1}^{\prime}$

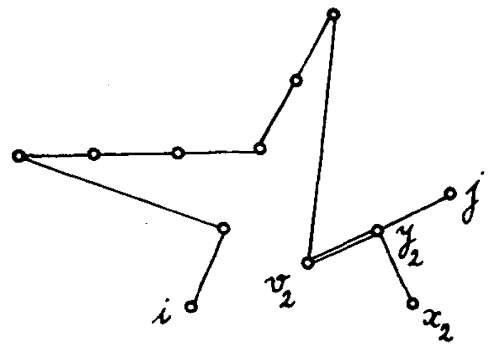

$T_{2}^{\prime}$

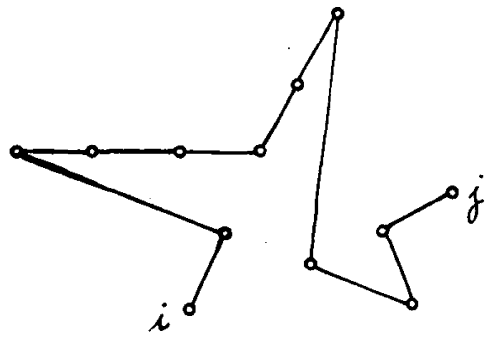

$T_{3}^{\prime} \equiv H P$
$T \equiv S S T$

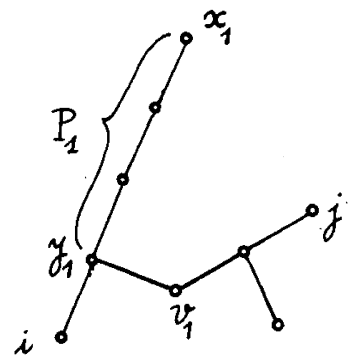

$T_{1}$

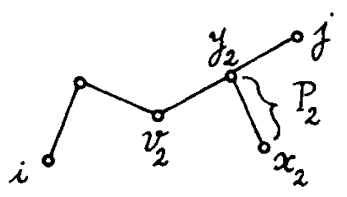

$T_{2}$

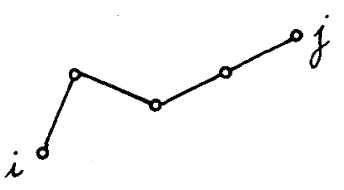

$T_{3} \equiv P_{3}$

vol. $17, \mathrm{n}^{\circ} 3$, août 1983

Figure 1 
Since $E(P) \cup E\left(P_{1}\right) \cup \ldots \cup E\left(P_{t-2}\right)$ is a partition of the edge set $E(T)$ of $T$ (see fig. 1), we can write:

$$
\sum_{i=0}^{t-3} c\left(P_{i}\right)=\sum_{i=0}^{t-2} c\left(P_{i}\right)-c\left(P_{t-2}\right)=c(T)-d_{T}(i, j)=c(T)-\operatorname{diam}(T),
$$

hence:

$$
c(\mathrm{HP}) \leqq 2 c(T)-\operatorname{diam}(T) .
$$

This heuristic algorithm for obtaining a SHP may proceed as follows: at each step is selected that terminal vertex $x \neq i, j$ such that the increase of the cost, equal to $c(x, v)-c\left(x_{p}, v\right)$ to be minimum.

THEOREM 1: If HP is a Hamiltonian path obtained with the preceding algorithm in the symmetric Euclidean case from $T$, which is a SST having $t$ terminal vertices, then:

$$
c(\mathrm{HP}) \leqq 2 c(T)-\operatorname{diam}(T) \leqq 2\left(1-\frac{1}{t}\right) c(T) .
$$

Proof: It remains to prove only that:

$$
2 c(T)-\operatorname{diam}(T) \leqq 2\left(1-\frac{1}{t}\right) c(T),
$$

or $\operatorname{diam}(T) \geqq(2 / t) c(T)$.

For this consider a terminal vertex $a \neq i, j$ of $T$, where $d_{T}(i, j)=\operatorname{diam}(T)$. There exists a unique path $a, a_{1}, a_{2}, \ldots, a_{r}, b$ in $T$ such that $b$ is the unique vertex of this path which belongs to the path between $i$ and $j$ in $T$ (see fig. 2).

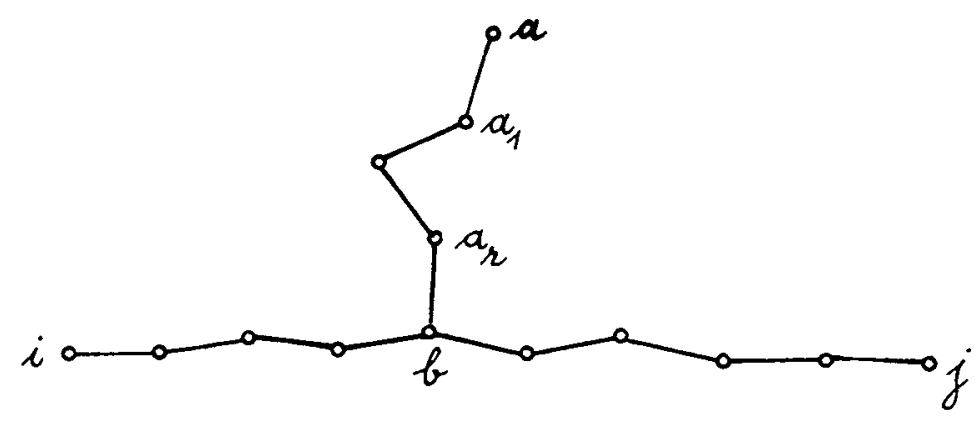

Figure 2

R.A.I.R.O. Recherche opérationnelle/Operations Research 
Since $d_{T}(i, j)=\operatorname{diam}(T)$ it follows that:

$$
d_{T}(a, b) \leqq d_{T}(i, b) \quad \text { and } \quad d_{T}(a, b) \leqq d_{T}(j, b),
$$

hence:

$$
2 d_{T}(a, b) \leqq d_{T}(i, b)+d_{T}(b, j)=\operatorname{diam}(T),
$$

or:

$$
d_{T}(a, b) \leqq \frac{1}{2} \operatorname{diam}(T) .
$$

Any edge not belonging to the path between $i$ and $j$ in $T$ joins two consecutive vertices of a path of the form $a, a_{1}, \ldots, a_{r}, b$ in $T$, where $a \neq i, j$ is a terminal vertex of $T$ and $b$ lies on the $i-j$ path in $T$. This implies:

$$
\begin{aligned}
c(T) \leqq d_{T}(i, j)+\sum_{a \neq i, j} d_{T}(a, b) & \\
& \leqq \operatorname{diam}(T)+\frac{t-2}{2} \operatorname{diam}(T)=\frac{t}{2} \operatorname{diam}(T),
\end{aligned}
$$

or:

$$
\operatorname{diam}(T) \geqq \frac{2}{t} c(T)
$$

and (7) is proved.

COROLlaRY 1: If SHP is a shortest Hamiltonian path in the symmetric Euclidean case and $T$ is a SST with $t$ terminal vertices, the following inequalities hold:

$$
c(\mathrm{SHP}) \leqq 2 c(T)-\operatorname{diam}(T) \leqq 2\left(1-\frac{1}{t}\right) c(T) .
$$

If the star $K_{1, n-1}$ is a spanning tree of $K_{n}$ such that the cost of any edge of $E\left(K_{1, n-1}\right)$ is equal to 1 and the cost of any edge of $E\left(K_{n}\right) \backslash E\left(K_{1, n-1}\right)$ is equal to 2 , the triangle inequality is verified.

In this case $K_{1, n-1}$ is a SST of $K_{n}, c(\mathrm{SHP})=2 n-4, c\left(K_{1, n-1}\right)=n-1$, $\operatorname{diam}\left(K_{1, n-1}\right)=2, t=n-1$ and all inequalities of (8) become equalities. This case is shown in figure 3 .

Now consider the spanning tree $T$ of $K_{n}$ composed from the path $1,2, \ldots, n-t+2$ and $t-2$ edges: $2, n-t+3 ; 3, n-t+4 ; \ldots ; t-1, n$, where $n \geqq 2(t-1), t \geqq 4$ such that the cost of any edge of $E(T)$ is equal to 1 and the vol. $17, \mathrm{n}^{\circ} 3$, août 1983 
cost of any edge $u v \in E\left(K_{n}\right) \backslash E(T)$ is equal to $d_{T}(u, v)$. An illustration is given in figure 4.

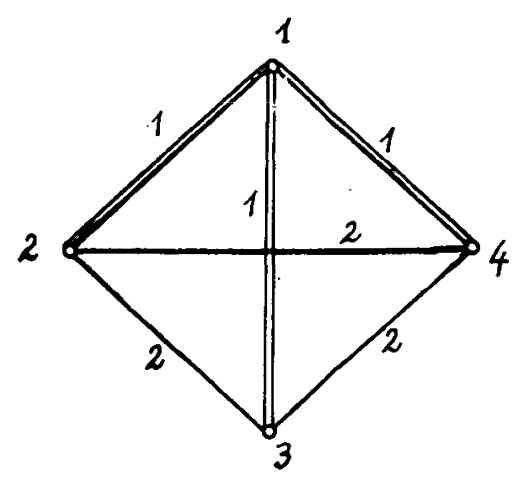

Figure 3

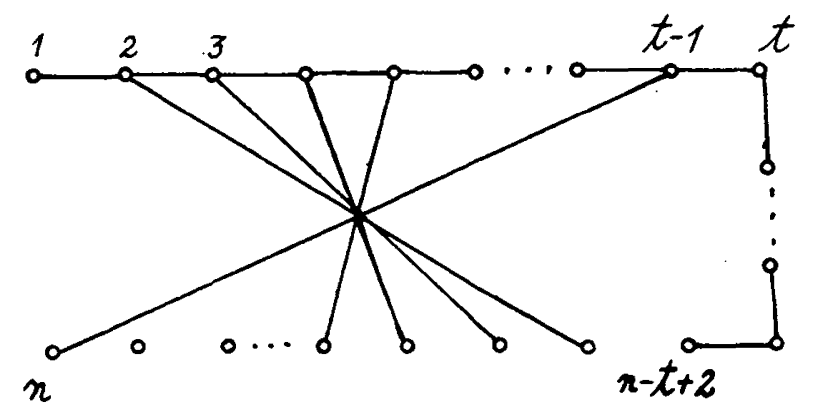

Figure 4

In this case the triangle inequality is satisfied, $T$ is a SST of $K_{n}$ having $t(T)=t$ terminal vertices and $c(T)=n-1, \operatorname{diam}(T)=n-t+1$. For a SHP of $K_{n}$ at least $t-2$ terminal vertices of $T$ are internal vertices, hence:

$$
c(\mathrm{SHP}) \geqq c(T)+t-2=n+t-3 .
$$

R.A.I.R.O. Recherche opérationnelle/Operations Research 
It follows that we can choose:

SHP: $1,2, n-t+3,3, n-t+4, \ldots, t-1, n, t, t+1, \ldots, n-t+2$, and $c(\mathrm{SHP})=n+t-3$.

We obtain:

$$
c(\mathrm{SHP})=2 c(T)-\operatorname{diam}(T)<2\left(1-\frac{1}{t}\right) c(T)
$$

since the inequality is equivalent to $n>t+1$, which is true because $n \geqq 2 t-2$ and $t \geqq 4$.

Note that if the proposed algorithm is applied to $T$ we find a SHP of $K_{n}$.

Corollary 2: If HP is a Hamiltonian path produced by the proposed algorithm in the symmetric Euclidean case from a SST having $t$ terminal vertices, it follows that:

$$
\frac{c(\mathrm{HP})}{c(\mathrm{SHP})} \leqq 2\left(1-\frac{1}{t}\right)
$$

This inequality results from (6) since $c$ (SST) $\leqq c$ (SHP).

D. Rosenkrantz, R. Stearns and P. Lewis [6] proved that in the symmetric Euclidean case if $T$ is a SST of $K_{n}$ then $c$ (SHC) $\leqq 2 c(T)$ (see also [2]). This result appears also as a consequence of the theorem 1:

CoRollary 3 (Rosenkrantz, Stearns, Lewis): If the costs satisfy triangle inequality then:

$$
c(\mathrm{SHC}) \leqq 2 c(T),
$$

where $T$ is a SST of $K_{n}$.

Proof: If HP is a Hamiltonian $i-j$ path obtained with the proposed algorithm, then adding edge $i j$ we find a $\mathrm{HC}$ for which the cost is equal to:

$$
c(i, j)+c(\mathrm{HP}) \leqq d_{T}(i, j)+2 c(T)-\operatorname{diam}(T)=2 c(T) .
$$

It follows that $c(\mathrm{SHC}) \leqq 2 c(T)$.

COROllary 4: If $\mathrm{HC}$ denotes the Hamiltonian cycle obtained in the symmetric Euclidean case from the Hamiltonian $i-j$ path HP by adding edge $i j$, then:

$$
c(\mathrm{HC}) \leqq 2 c(T) \leqq 2\left(c(\mathrm{SHC})-c\left(e_{\max }\right)\right)
$$

where $T$ is the SST from which $\mathrm{HP}$ is deduced and $e_{\max }$ denotes an edge of SHC having maximum cost. 
Proof: By the removal of the edge $e_{\max }$ of SHC we get a HP, which is a spanning tree of $K_{\mathrm{m}}$, hence:

$$
c(T) \leqq c(\mathrm{SHC})-c\left(e_{\mathrm{max}}\right)
$$

Note that this upper bound for the cost of HC is the same as for the Hamiltonian cycle obtained by the nearest insertion rule (NIR procedure of [4]), proposed by D. Rosenkrantz, R. Stearns and P. Lewis [6].

From corollary 4 it follows also that:

$$
\frac{c(\mathrm{HC})}{c(\mathrm{SHC})} \leq 2-\frac{1}{n}
$$

since:

$$
c\left(e_{\max }\right) \geq \frac{c(\mathrm{SHC})}{n} \text {. }
$$

\section{ACKNOWLEDGEMENTS}

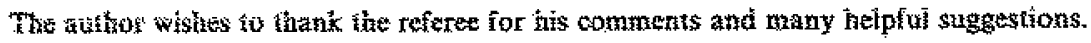

\section{REFERENCES}

1. M. Behzad, G. Chartrand and L. Lesniak-Foster, Graphs and Digraphs, Prindle, Weber and Schmidt, Boston, Massachusetts, 1979.

2. 1. A. Bonoy and U. S. R. MuRTY, Graph Theory tqith Applications, The Macmillan Press, London, 1977.

3. N. Chastonses, The Shortest Hamiltonian Chain of a Graph, S.I.A.M. J. Appl. Math. Vol. 19, 1970, pp. 689-696.

4. N. Christoribes, The Travelling Salesman Problem, Combinatorial Optimization, John Wiley, 1979, pp. 131-149.

5. M. HEed and R. M. KARP, The Tranelling-Solesman Problem and Mintmum Spanning Trees, Operations Research, Vol. 18, 1970, pp. 1138-1162.

6. D. I. Rospmkantz, R. E. STenkNs and P M. Lewts, An Anatysis of Several Heuristics for the Trateling Salesman Problem, S.I.A.M. J. Comput., Vol 6, No. 3 , 1977, pp. $\$ 63-\$ 81$.

7. I. ToMescti, Un algorithme pouf Fobtention d"une choîte homiltonienne en partant de Parbre mintimal a un graphe, R.A.I.R.O., V.3, Vol. 9, 1975, pp. 5-12, 